\title{
Brachytherapy in cervical cancer radiotherapy during COVID-19 pandemic crisis: problems and prospects
}

\author{
Dr. Raviteja Miriyala, MD, Umesh Mahantshetty, MD, DNB \\ Department of Radiation Oncology. Homi Bhabha Cancer Hospital and Research Centre, Visakhapatnam, Andhra Pradesh, India
}

\begin{abstract}
COVID-19 pandemic has adversely affected general healthcare delivery systems and cancer care throughout the world. The management of locally advanced cervical cancer presents specific challenges in this context, especially brachytherapy and completion of radiotherapy treatment, without compromising the overall treatment time and anticipated outcomes. This article presents in detail the issues and possible solutions with currently available literature for COVID-19 and radiation, in particular brachytherapy management of locally advanced cervical cancers. The review attempts to provide possible explanations and pathways based on COVID-19 testing, brachytherapy processes including application, imaging, and fractionation, keeping in mind the overall treatment time.
\end{abstract}

J Contemp Brachytherapy 2020; 12, 3: 290-293 DOI: https://doi.org/10.5l14/jcb.2020.96873

Key words: COVID-19, cervical cancer radiotherapy, brachytherapy, HDR fractionation.

\section{Purpose and rationale}

The COVID-19 pandemic has adversely affected healthcare delivery systems throughout the world [1] While the impact of this ongoing pandemic is largely asymmetric, with relatively developed countries bearing the largest burden of the virus, developing economies with less robust infrastructure are still bracing for the peak of the pandemic. Healthcare in general and cancer care particularly, are still evolving to adapt to this unprecedented challenge. Also, this crisis has caused the entire community to reconsider and revisit potential strategies for maintaining the safety of health caregivers, patients, and establishments. Cancer management poses a unique challenge in terms of a long duration of treatment, a need for regular monitoring, acute and delayed morbidities associated with aggressive therapies, etc., resulting in an enhanced risk of contracting infections [2]. Nevertheless, cancer treatment has been declared as an essential service that cannot be compromised during the pandemic $[3,4]$. Numerous guidelines and recommendations have been published by various agencies regarding the management of malignancies under these unusual circumstances $[5,6]$.

The management of locally advanced cervical cancer presents specific challenges in this context. It is a rapidly proliferating cancer with high cure rates; therefore, postponement of treatment is an unviable option $[7,8]$. External beam chemo-radiotherapy, which is the standard treatment, is usually delivered in conventional fractions of $1.8 \mathrm{~Gy}$ to $2 \mathrm{~Gy}$, over 4.5 to 5 weeks. Unlike other malignancies (e.g., prostate or lung), hypofractionated regimes to reduce the treatment time have not been proven to be efficacious in cervical cancer.

Brachytherapy for curative management of cervical cancer is indispensable, usually follows chemo-radiotherapy, and accounts for $50 \%$ of the radiation treatment [9]. Brachytherapy is delivered in multiple applications and fractions, with each application requiring placement of intracavitary/interstitial applicators under anesthesia, followed by imaging, planning, and treatment delivery. Therefore, brachytherapy may portend additional risks to the patient as well as the health caregivers in the time of pandemic. Hence, all processes related to brachytherapy, such as application, imaging, planning, and delivery described in various guidelines need to be adapted to a pandemic environment [10]

\section{COVID-19 testing and triage}

Before brachytherapy, along with routine pre-procedure work-up, the COVID-19 testing should be considered before each application. For patients who are hospitalized for the entire duration of brachytherapy, single testing before the first application may be considered sufficient, if appropriate precautions are assured during their hospital stay. This has the potential to reduce highrisk exposure to health caregivers, especially since early studies have identified the presence of coronavirus in 
urine and anal swabs in asymptomatic individuals apart from droplet borne spread $[8,11]$. However, the accuracy of testing modality used should be taken into account. For patients who were admitted and tested negative for COVID-19 during external beam radiotherapy, the extension of hospital admission to complete brachytherapy in a relatively safe environment may be considered to limit the need for repeated testing. However, in areas with a high incidence of COVID-19, tests may be repeated before each application, with an assumption that a negative test is 'valid' for 3 to 5 days. Figure 1 shows schema and possible workflow for cervical cancer patients and COVID testing for brachytherapy. Patients who test negative for COVID-19 should be treated with universal precautions, to reduce potential high-risk exposures to patients and the staff. For those who have suspicious symptoms, or for those with equivocal results on initial testing, deferring brachytherapy, isolation, and prompt (re) testing depending on institutional policies to confirm COVID-19 status may be a reasonable option before the further course of action is decided.

For patients who test positive, symptomatic or asymptomatic, the management strategy is more complicated and involves critical decisions for treatment of COVID-19, the continuation of treatment with brachytherapy, protection of health caregivers involved in brachytherapy processes and delivery, and other routine processes. Though evidence-based recommendations for the management of COVID-19 are available in the current international guidelines, questions regarding the management of cancer in patients who test positive before or during their cancer treatment remain mostly unanswered $[12,13,14,15]$. This mainly applies to the delivery of radiation therapy in cervical cancer, where overall treatment time is an important prognostic factor $[10,16]$. Some small, retrospective series have doc- umented increased peri-operative mortality and morbidity in COVID-19 positive patients, who underwent major elective and emergency surgeries $[14,15,17]$. However, data on minor procedures including brachytherapy, is lacking at present. While the risks of continuing brachytherapy surely outweigh the benefits in symptomatic positive patients, strategies for treating asymptomatic positive patients need to be resource-customized. Employing a committed team, with a full gear of protective equipment, dedicated operating room and treatment machine, disinfection protocols for brachytherapy equipment, etc., may not be a feasible option for most centers [18]. In such circumstances, it is not unreasonable to wait for the recovery of patient and a negative COVID-19 result. Once a negative result is documented, re-testing may be done after a week (or as recommended by the local public health authorities) and brachytherapy can be considered accordingly. However, the number of applications ought to be limited and delivering multiple fractions per each application should be considered in such cases to compensate the unnecessary prolongation in overall treatment time. Maintaining a fine balance between the potential risks associated with such attempts and the risks related to undue prolongation of overall treatment time is imperative for achieving optimal outcomes. In principle, cancer care units should be well-prepared to confront such situations, with appropriately equipped ICU facilities and advanced precautions and measures.

\section{Brachytherapy application, anesthesia, and imaging}

Pre-operative parts preparation, medications, and consenting for brachytherapy procedures should include disclaimers related to the risk of COVID-19 infection. Pre-operative admissions may be avoided to reduce the

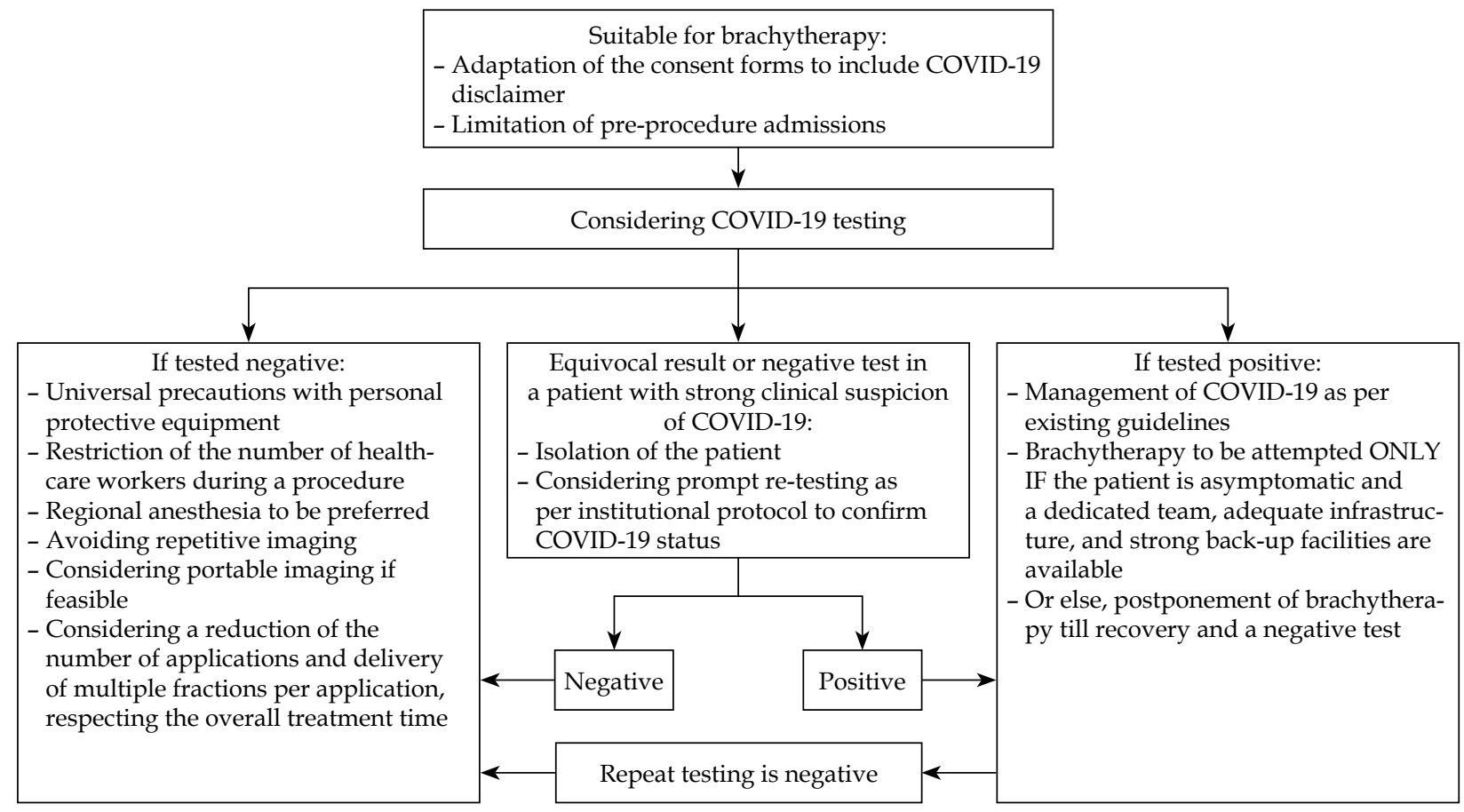

Fig. 1. Schema and possible workflow for cervical cancer patients and COVID-19 testing for brachytherapy 
risk of acquiring infection from asymptomatic patients in general wards; alternatively, admission in isolated rooms may be considered.

During the brachytherapy procedure, universal precautions with a personal protective equipment and appropriate disinfection practices should be absolutely adopted. Similarly, the number of health caregivers needed for the procedure should be restricted to the bare minimum [8]. Wherever applicable, the staff should be divided into groups, so that ongoing treatments can be sustained even if some of the members are exposed or infected.

Regional anesthesia should be preferred wherever feasible, to avoid aerosol-generating procedures associated with general anesthesia. However, achieving adequate perineal and pelvic muscle relaxation for vaginal packing assume even greater importance, to stabilize the application for longer durations and reduce the doses to organs at risk, especially if larger dose per fraction or multiple fractions per application are planned [16,19].

Repetitive imaging for treatment planning may pose additional risks to patients and health caregivers. The use of portable imaging in the brachytherapy treatment area to restrict multiple patient transfers should be implemented. If resources for imaging are limited, 2-dimensional planning can be considered, with point A-based prescriptions. For facilities practicing volumetric planning, the use of MRI planning can be restricted to the first application, mainly if the volume of residual disease is low and extensive reduction in further fractions is not anticipated. Alternatively, CT and trans-rectal ultrasound can also be used for volumetric planning in experienced facilities [10]. In centers where resources for planning are severely restricted, library plans available with the treatment planning system can be used based on applicator parameters, if the geometry is well-maintained and reproducible.

\section{Dose, fractionation, and application}

Delivery of multiple fractions per application, ranging from 2-5, has the potential to limit high-risk exposures to health caregivers and patients. However, such attempts should be based on sound and robust biological rationale, and supported by clinical evidence, so that appropriate balance can be achieved between high-risk exposures and disease/toxicity-related outcomes, without undue compromise of either. Needless to say, clinical factors like site and volume of residual disease, type of brachytherapy application, imaging modalities utilized, implant geometry, relative doses to the organs at risk, logistics of treatment facility, accessibility, expected compliance, etc., should be considered before adapting the recommended fractionation schedules [10]. Table 1 presents various fractionation schedules, number of applications, biological equivalent doses, and specific pros and cons remarks for each published $[16,20,21,22]$.

The American Brachytherapy Society recommends five to six fractions of 5-6 Gy each, interdigitated with external beam radiation [20]. While this radio-biologically sound schedule and has a strong evidence base a strong evidence base for outcomes, it is limited by the number of necessary applications. International multicenter studies by the EMBRACE group have reported excellent local control and toxicity outcomes with four fractions of $7 \mathrm{~Gy}$, delivered in 2 brachytherapy applications over 1 week, with 2 fractions per application in the majority [16,23]. The additional advantage of this regimen is that applications can be limited to two, without compromising the number of fractions. Another attractive option is to deliver high doses per fraction for each application. Retrospective and prospective studies have shown larger fraction sizes of 9 Gy in two applications to have inferior local control without a significant impact on overall survival $[20,21]$. However, such regimes can still be considered for small volume residual or elderly patients, after the assessment of risks and benefits.

Single application with multiple fractions has been practiced for pure interstitial template-based brachytherapy boost in post-operative recurrences, with reasonably good outcomes [24]. Currently, there have been attempts to deliver multiple fractions in a single application for intact cervical cancer, where MRI-based planning was used and 9 Gy was delivered on day 1 , and two fractions

Table 1. Various fractionation schedules, number of applications, biological equivalent doses, and specific pros and cons remarks for each published

\begin{tabular}{|c|c|c|c|c|}
\hline Schedule & $\begin{array}{l}\text { No. of } \\
\text { applications }\end{array}$ & $\begin{array}{l}\mathrm{EQD}_{2} \text { doses for } \\
\text { tumor in } \mathrm{Gy} \\
(\alpha / \beta-10) \text { for } \mathrm{BT}\end{array}$ & Remarks & Reference \\
\hline $5-6 \mathrm{~Gy} \times 5-6$ fractions & $5-6$ & $37.5-40$ & $\begin{array}{c}\text { Increased risk of exposure due to large } \\
\text { number of applications }\end{array}$ & [20] \\
\hline 7 Gy $\times 4$ fractions & 2 & 39.7 & $\begin{array}{l}\text { Limited applications, without compromis- } \\
\text { ing the number of fractions }\end{array}$ & [16] \\
\hline 8 Gy $\times 3$ fractions & 3 & 36 & $\begin{array}{l}\text { Overall treatment time may be prolonged } \\
\text { if not interdigitated with EBRT }\end{array}$ & {$[22]$} \\
\hline 9 Gy $\times 2$ fractions & 2 & 28.5 & $\begin{array}{c}\text { Compromised local control, especially for } \\
\text { large volume residual }\end{array}$ & {$[20,21]$} \\
\hline $\begin{array}{l}9 \text { Gy (on day } 1 \text { ) followed } \\
\text { by } 7 \text { Gy } \times 2 \text { fractions } \\
\text { (on day } 2 \text {, delivered } \\
\text { at least } 6 \text { hours apart) }\end{array}$ & 1 & 34.1 & $\begin{array}{l}\text { Risk of exposure reduced, logistic advan- } \\
\text { tage; early outcomes encouraging } \\
\text { Long-term outcomes awaited }\end{array}$ & $\begin{array}{l}\text { Unpublished (CTRI/2017/ } \\
\text { 03/008172, SIMBRACE } \\
\text { study, Tata Memorial } \\
\text { Hospital, Mumbai) }\end{array}$ \\
\hline
\end{tabular}


of 7 Gy were delivered on day 2, after confirmatory CT imaging to review and address inter-fraction variations (CTRI/2017/03/008172, SIMBRACE study, Tata Memorial Hospital, Mumbai; abstract accepted for WCB 2020). Even though the feasibility of such regimen has been proven in that study and early disease-related outcomes are encouraging, long-term results regarding toxicity are awaited. Finally, at brachytherapy plan evaluation, if multiple applications are considered necessary to maintain a favorable therapeutic ratio, the brachytherapy applications can be repeated at shorter intervals (twice a week), on a case-by-case basis, to achieve and maintain the optimal overall treatment time.

In summary, the restriction of pre-operative admissions, mandatory pre-procedure testing for COVID-19, triaging patients and appropriate management according to test results, adherence to universal precautions, the use of regional anesthesia, reducing the number of applications, and delivering multiple fractions with each application, are some of the practical alternatives that can be explored for delivering essential brachytherapy treatments for cervical cancer patients, without unduly compromising the therapeutic ratio. Such measures should be individualized and customized to the severity of the pandemic in each region, to limit high-risk exposures to patients, health caregivers, and establishments/institutions in the future.

\section{Disclosure}

The authors report no conflict of interest.

\section{References}

1. Emanuel EJ, Persad G, Upshur R et al. Fair allocation of scarce medical resources in the time of Covid-19. N Engl J Med 2020; 382: 2049-2055.

2. Wang H, Zhang L. Risk of COVID-19 for patients with cancer. Lancet Oncol 2020; 21: e181.

3. https://www.who.int/publications-detail/covid-19-operational-guidance-for-maintaining-essential-health-services-during-an-outbreak

4. https://www.mohfw.gov.in/pdf/EssentialservicesduringCOVID19updated0411201.pdf

5. Al-Shamsi HO, Alhazzani W, Alhuraiji A et al. A practical approach to the management of cancer patients during the novel coronavirus disease 2019 (COVID-19) pandemic: an international collaborative group. Oncologist 2020; 25 : e936-e945.

6. Burki TK. Cancer guidelines during the COVID-19 pandemic. Lancet Oncol 2020; 21: 629-630.

7. https://www.americanbrachytherapy.org/about-abs/absnews/abs-statement-on-coronavirus/

8. Roussy G, Chopra S, Viswanathan A et al. Adv Radiat Oncol (In press).

9. Holschneider $\mathrm{CH}$, Petereit DG, Chu C et al. Brachytherapy: a critical component of primary radiation therapy for cervical cancer: from the Society of Gynecologic Oncology (SGO) and the American Brachytherapy Society (ABS). Brachytherapy 2019; 18: 123-132.

10. Mahantshetty U, Gudi S, Singh R et al. Indian Brachytherapy Society Guidelines for radiotherapeutic management of cervical cancer with special emphasis on high-dose-rate brachytherapy. J Contemp Brachytherapy 2019; 11: 293-306.
11. Peng L, Liu J, Xu W et al. SARS-CoV-2 can be detected in urine, blood, anal swabs and oropharyngeal swabs samples. J Med Virol 2020; 10.1002/jmv.25936.

12. https://www.who.int/emergencies/diseases/novel-coronavirus-2019/technical-guidance/patient-management

13. Ramirez PT, Chiva L, Eriksson AGZ et al. COVID-19 global pandemic: options for management of gynecologic cancers. Int J Gynecol Cancer 2020; 30: 561-563.

14. https://www.bgcs.org.uk/wp-content/uploads/2020/03/ BGCS-covid-guidance-v1.22.03.2020.pdf

15. https://www.facs.org//media/files/covid19/guidance for_triage_of_nonemergent_surgical_procedures.ashx

16. Tanderup K, Fokdal LU, Sturdza A et al. Effect of tumor dose, volume and overall treatment time on local control after radiochemotherapy including MRI guided brachytherapy of locally advanced cervical cancer. Radiother Oncol 2016; 120: 441-446.

17. COVID Surg Collaborative. Global guidance for surgical care during the COVID-19 pandemic. Br J Surg 2020; 10.1002/ bjs. 11646 .

18. https://www.estro.org/About/Newsroom/News/Radiotherapy-in-a-time-of-crisis

19. Patel FD, Kumar P, Karunanidhi G et al. Optimization of high-dose-rate intracavitary brachytherapy schedule in the treatment of carcinoma of the cervix. Brachytherapy 2011; 10: 147-153.

20. Albuquerque K, Hrycushko BA, Harkenrider MM et al. Compendium of fractionation choices for gynecologic HDR brachytherapy - An American Brachytherapy Society Task Group Report. Brachytherapy 2019; 18: 429-436.

21. Hendry J, Jones GW, Mahantshetty UM et al. Radiobiological analysis of outcomes using external beam radiotherapy plus high dose-rate brachytherapy (4x7 Gy or 2x9 Gy) for cervical cancer in a multi-institution trial. Int J Radiat Oncol Biol Phys 2017; 99: 1313-1314.

22. Rao BS, Das P, Subramanian BV et al. A comparative analysis of two different dose fractionation regimens of high dose rate intracavitary brachytherapy in treatment of carcinoma of uterine cervix: a prospective randomized study. J Clin Diagn Res 2017; 11: XC06-XC10

23. Mahantshetty U, Krishnatry R, Hande V et al. Magnetic resonance image guided adaptive brachytherapy in locally advanced cervical cancer: an experience from a tertiary cancer center in a low and middle income countries setting. Int J Radiat Oncol Biol Phys 2017; 99: 608-617.

24. Mahantshetty U, Shrivastava S, Kalyani N et al. Templatebased high-dose-rate interstitial brachytherapy in gynecologic cancers: a single institutional experience. Brachytherapy 2014; 13: 337-342. 